\title{
Implementation of NSOM to Biological Samples
}

\author{
S. Prauzner-Bechcicki ${ }^{a, b}$, J. Wiltowska-Zuber ${ }^{a}$, A. Budkowski ${ }^{b}$, M. LekKa $^{a}$ \\ AND J. RYSZ ${ }^{b, *}$ \\ ${ }^{a}$ The Henryk Niewodniczański Institute of Nuclear Physics, Polish Academy of Sciences \\ E. Radzikowskiego 152, 31-342 Kraków, Poland \\ ${ }^{b}$ The Smoluchowski Institute of Physics, Jagiellonian University, W.S. Reymonta 4, 30-054 Kraków, Poland
}

\begin{abstract}
Near-field scanning optical microscopy is a technique providing images of structures with spatial resolution better than $\lambda / 2$, which is undetectable in far-field where the Abbe law of limiting resolution is critical. In parallel to the optical imaging, topography maps are also acquired. Near-field scanning optical microscopy measurements can be performed both in air and liquid environments. The later makes the technique very useful for biomaterials analysis offering information that could not be obtained with other methods. Our work presents the results of recent studies on application of near-field scanning optical microscopy to imaging of cells in air as well as in physiological buffers. Differences in cell's topography and morphology have been noticed between two cell lines from human bladder non-malignant (HCV29) and malignant (T24) cancers. Presented results are part of the research that characterizes physiological changes of cells depending on stage of cancer.
\end{abstract}

PACS: 87.64.M-, 87.64.mt

\section{Introduction}

Significant increase of microscope methods, based on a scanning probe, was noticed in recent three decades [1-7]. One of them, next to atomic force microscopy (AFM [4]), is near-field scanning optical microscopy (NSOM $[2,7]$ ). In both techniques, the measurements can be performed both in air and liquid environments in a noninvasive way. When applied in studies of biological samples, this technique might bring new information, which, in many cases, cannot be obtained by other standard techniques [8-10].

In research focused on properties of biological materials, the NSOM technique gives a possibility to detect structures with sizes below $\lambda / 2$ that are not detectable in a far-field where the Abbe criterion is preserved. In such a case, the NSOM microscope can be a very useful tool due to its high-resolution especially when combined with fluorescent techniques. The latter one in conjunction with labeling methods could bring information about distribution of molecules of interest like, for example, surface receptors present in a cellular membrane $[2,11]$. Moreover, the measurements of optical properties in liquid environments will give the opportunity to observe changes of living cells in their natural environment [12].

Despite that, NSOM measurements in liquid environment are still not widely applied to biological objects $[2,11-14]$. This is due to a strong interaction of the

\footnotetext{
* corresponding author; e-mail: jakub.rysz@uj.edu.pl
}

scanning probe with the surface of the studied soft materials.

In this paper, we present the results of NSOM measurements carried out on fixed cells taken in ambient and liquid conditions. The aim of the study was to evaluate changes of optical properties of biological samples during drying process. Differences in cellular topography and morphology have been noticed between two cell lines from human bladder non-malignant (HCV29) and malignant (T24) cancers as well. The obtained NSOM images were compared with those recorded by AFM i.e. topography images.

\section{Materials and methods}

\subsection{Cell lines}

The studies were performed on two human cell lines: reference HCV29 (non-malignant transitional epithelial cells of the urether, ATCC) and T24 (transitional cell cancer of the urine bladder, ATCC). Cells were grown in culture flasks (Saarstedt) in a RPMI 1640 medium (Sigma) supplemented with $10 \%$ foetal calf serum (Sigma, pH 7.4) and with 1\% mixture of antibiotics (streptomycin, neomycin and penicillin, Sigma), at $37^{\circ} \mathrm{C}$ in $95 \%$ air $/ 5 \% \mathrm{CO}_{2}$ atmosphere. After few passages, cells were trypsinized using $0.05 \%$ in EDTA-trypsin solution (Sigma), transferred to the Petri dishes with a coverslip inside, and cultured until semiconfluent layer was formed. Afterwards, they were fixed and dried when needed for NSOM measurements or left alive for AFM imaging. 


\subsection{Cell fixation}

Prior to measurements, cells were fixed either with $2 \%$ (for NSOM measurements in liquid) or $3.7 \%$ (NSOM in air) paraformaldehyde solution in PBS (phosphate buffered saline, pH 7.4, Sigma). First, the culture medium was soaked out from the Petri dish. Then, the coverslip was rinsed few times with the PBS buffer only. A fixative solution of paraformaldehyde was added for 12 min at room temperature. Afterwards, the solution was moved out from the coverslip, followed by rinsing it few times with the PBS buffer only.

\subsection{Drying procedure}

To perform NSOM experiments in ambient conditions, the sample had to dry out. Therefore, after the fixation, each sample was successively washed with the PBS buffer dissolved in water at the concentrations of 1:2, 1:5, 1:10, and finally washed out with deionized water only (Cobrabid purification systems, $18 \mathrm{M} \Omega \mathrm{cm}$ ). Such protocol of drying of samples provided no crystallization of salt on the sample surface, and kept cell membrane unharmed during the applied way of dehydratation.

\subsection{NSOM measurements}

To overcome the diffraction criterion that limits resolution of standard microscopy, so called far-field microscopy, a light source should be brought very close to sample surface. When the distance between light source and studied surface is smaller than wavelength of incident light, the diffraction limits do not occur. In this regime, so called near-field, the resolution is limited by the size of the light source only. To achieve this a light from a laser is delivered into an optical fiber. Other end of the fiber is tempered to form a sharp tip with aperture ranging from tens to hundreds of nm. At the aperture with sub-wavelength diameter, usually from $50-250 \mathrm{~nm}$, an evanescent wave is generated next to the total internal reflection of light. The evanescent wave can interact with the surface to reconstruct as a propagating wave that is detectable in the far-field, delivering information about structures of a material placed in near-field [15].

To perform the NSOM experiment, a point light source should be brought close to the investigated surface and then a scan is performed. An optical signal coming from the surface is collected and detected over (reflection) or below (transmission) the measured surface (Fig. 1). The diagram of NSOM microscope is shown in Fig. 2.

The topography and NSOM images were recorded using a commercially available device (MV1000, Nanonics), which is equipped with the "liquid cell" setup. The studies were performed in two modes: reflection and transmission ones. Standard NSOM aperture probes (Nanonics) were used with the aperture diameter of the $50 \mathrm{~nm}$ and $100 \mathrm{~nm}$. As a light source, a green laser with wavelength of $532 \mathrm{~nm}$ and power of $20 \mathrm{~mW}$ (produced by Lambda-Pro) was employed. Typical scan of $256 \times 256$ points was performed. This corresponds to square of $50 \mu \mathrm{m} \times$ a)

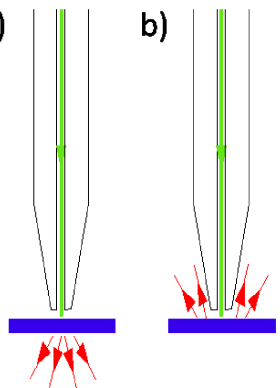

Fig. 1. Illustrations of two types of NSOM measurement modes: transmission (a), in which the photomultiplier is placed below the sample, and reflection (b) where light is detected above the sample.

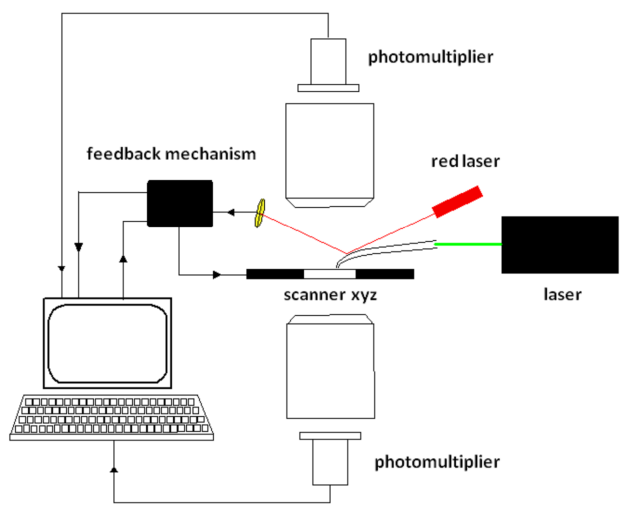

Fig. 2. Diagram of NSOM microscope.

$50 \mu \mathrm{m}$. In each point, the signal was recorded during $10 \mathrm{~ms}$.

Distance between the tip and the surface is controlled by a feedback mechanism similar to that used in non-contact AFM experiments. In this mode, the NSOM probe oscillates at a certain frequency close to the resonant one, which is usually in the range $50-150 \mathrm{kHz}$. Since the resonant frequency is a frequency where the probe has a tendency to oscillate with the largest amplitudes, any changes in the tip-surface distance will cause both shifts of the frequency value and reduction of the amplitude. The signal of the modified amplitude is sent to feedback system, which uses it to adjust the distance between tip and the investigated surface. In parallel, the same changes in amplitude signal are a basis for obtaining the topography image of the sample (similarly as in the AFM system).

A "liquid cell" setup allows to perform measurements in a liquid environment. Sample is mounted inside the bath. To control the tip position, two mirror setups are used (Fig. 3).

\subsection{AFM measurements}

The topography of sample surface was recorded using commercially available atomic force microscope Agilent 5500. Measurements were carried out in liquid en- 


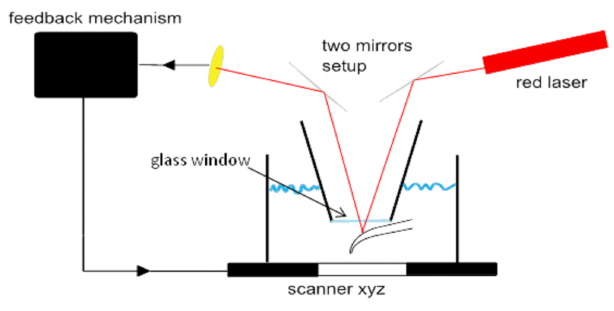

Fig. 3. The idea of MV1000 NSOM "liquid cell" setup with two mirrors in the beam bounce feedback system.

vironment using "liquid-cell" setup. The magnetic AC, non-contact mode (so called MAC mode) was employed with the scan rate of $1 \mathrm{~Hz}$. Standard MAC levers for experiments in liquid were used (Agilent, MAC lever type VI). Spring constant was $0.292 \mathrm{~N} / \mathrm{m}$ and the nominal resonant frequency in air was $66 \mathrm{kHz}$. The lateral resolution of images were collected as squares composed of $512 \times 512$ points.

\section{Results and discussion}

\subsection{AFM topography in liquid}

To determine the morphological features of the investigated cells, the topography images were collected using the AFM working in non-contact mode. This mode of operation has been chosen in order to compare the images with those recorded using NSOM (which also works in non-contact mode). The HCV29 line contains cells originating from non-malignant transitional epithelial cells of the urether. They have elongated shape with a length (along long axis) up to $100 \mu \mathrm{m}$ and height up to few $\mu \mathrm{m}$. In Fig. 4, the resulting AFM topography and phase are shown. T24 cells (transitional cancer cells of the urine bladder, Fig. 5) reach similar heights but their shape is rather oval with diameter up to $50 \mu \mathrm{m}$. For both cell lines, the phase images show that the surface of cells is not smooth but possesses some details on the surface clearly visible (phase images) for both cell lines.
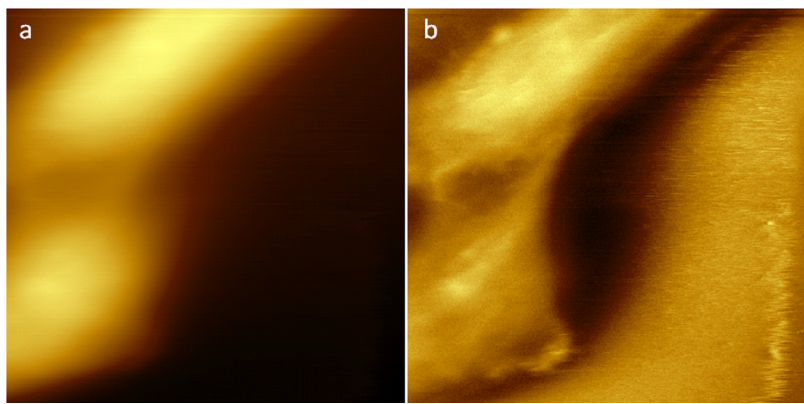

Fig. 4. Topography (non-contact mode) and phase images obtained for HCV29 cells in liquid conditions using $\operatorname{AFM}((\mathrm{a})$ and (b), respectively, scan size $50 \mu \mathrm{m} \times$ $50 \mu \mathrm{m})$.
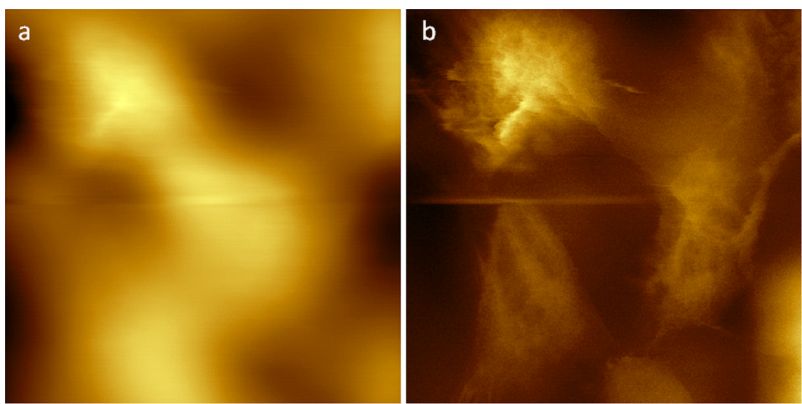

Fig. 5. Non-contact topography and phase images of T24 cells recorded in liquid conditions using AFM ((a) and (b), correspondingly, scan size $60 \mu \mathrm{m} \times 60 \mu \mathrm{m})$. The horizontal line visible both in topography and phase image is an artifact caused by a small particle adhered temporarily to the tip.

\subsection{NSOM imaging in air}

First, the NSOM experiments were carried out for dried cells in ambient conditions. To do that, after fixation of cells with $3.7 \%$ paraformaldehyde, a drying protocol has been applied according to protocol described in Sect. 2. Such prepared samples were measured using two modes of NSOM operation: transmission and reflection. Depending on the type of imaged cells either transmission or reflection mode was chosen. The HCV29 cells showed better images in reflection while T24 ones in transmission.

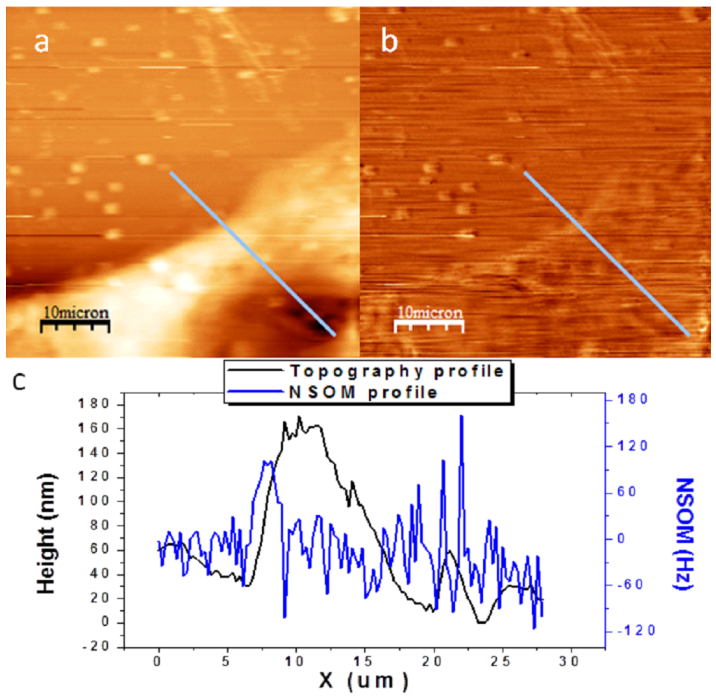

Fig. 6. HCV29 cells in air: (a) topography (non-contact mode), optical (b) images (reflection mode), and profile (c) obtained along a selected line.

Figure 6 presents an exemplary image of non-malignant HCV29 cells recorded in reflection mode. The topography shows the fragment of an elongated cell which is in agreement with AFM image of surface topography recorded for these cells. The NSOM image collected in 
the reflection mode presents almost no signal coming from the cell - the signal from the region without a cell (a glass coverslip) was almost the same as that from the cell. The comparison between the height and NSOM profiles (Fig. 6c) shows that oscillations of NSOM signal (on average $\approx 0$ ) do not correspond to the height signal, i.e. there is no significant difference depending whether it comes from glass or cell surfaces, even at the highest part of the profile. However, one can see such correlation at the border between glass and cell. The optical properties at this location have a large NSOM signal $(140 \mathrm{~Hz})$.

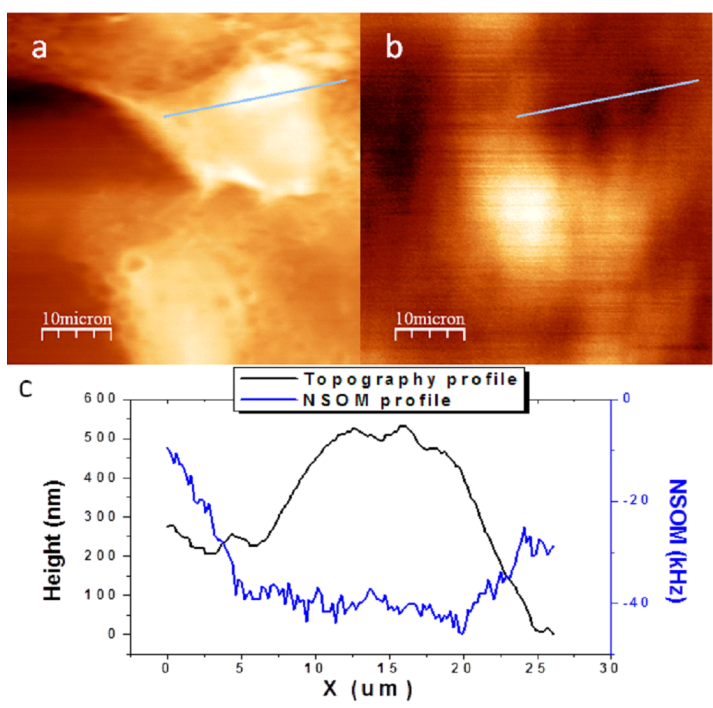

Fig. 7. T24 cells in ambient conditions: topography (a), optical (b) images (transmission mode), and profile (c) along the selected line.

The T24 cells recorded in transmission mode show the local increase of the optical density (Fig. 7b). By comparing it with the topography image (Fig. 7a), one can point out that the localization of the denser biological material like nucleus is possible. To study the correlation between the sample height and NSOM signal, profiles along the selected line were obtained (Fig. 7c). For T24 cells, the decrease of the NSOM signal was accompanied by the increase of the measured height. This can be explained by the fact that in transmission mode, the thicker sample transmits less light that can be collected by the photomultiplier due to the higher probability of the reflection and deflection of incident light.

The interaction between the probe and measured surface is well controlled so the stability of these types of measurements is good. The problem is that for many biological materials, especially living cells, the ambient conditions are not the natural ones. The drying procedure removes water from inside of the cell, which changes the structure of membrane and also influences strongly on internal organization of cellular components. Therefore as a next step, the NSOM imaging was performed in liquid conditions.

\subsection{NSOM imaging in liquid}

To elaborate the capability of NSOM imaging in liquid conditions, the HCV29 and T24 cells were examined. In liquid, the interaction between the measured surface and the tip end is much harder to control due to the strong dependence of the amplitude of the oscillating probe on the distance between the tip and the surface. It manifests in the fact that the choice of the frequency is much different from the resonant one. This is leading to incorrect feedback control resulting in both bad quality of the images and large error (at the limit, this can cause crashing probe and the damage of the sample). To determine the frequency of the tip oscillations suitable for imaging, the tip should oscillate within certain range of frequencies.

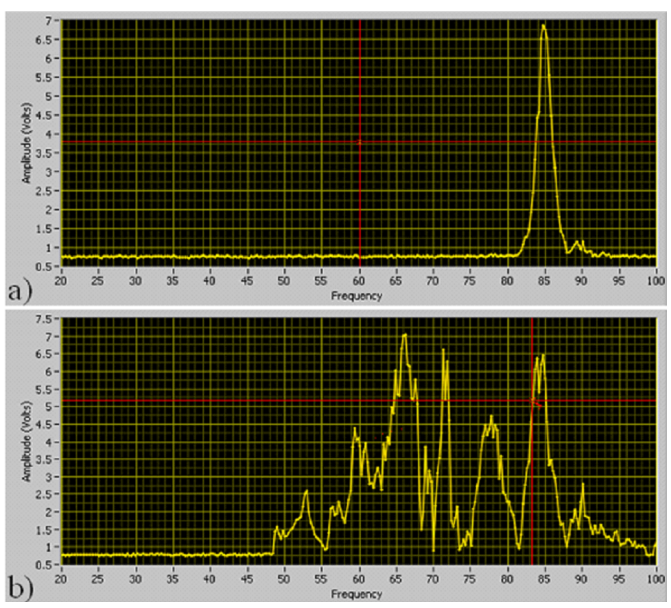

Fig. 8. Frequency spectra of the similar NSOM probe recorded in ambient (a) and liquid (b) conditions, within the same range of frequencies.

Figure 8 shows the frequency spectrum collected in air and liquid (water) conditions using the same type of the NSOM probes (SuperSensor NSOM/AFM Probe $\mathrm{Cr} / \mathrm{Au}$, Nanonics), oscillating at the similar range of frequencies. Additional oscillations, visible in Fig. 8b, are caused by the change of the environment - from air to water. The amplitude of the oscillations in liquid environment dropped down. This is the reason why the coercive force should be enhanced simultaneously leading to the increase of oscillations that are not resonant in origin. This makes the selection of the correct frequency and distance control more difficult.

To image the surface of fixed cells, the resonant frequency of $55 \mathrm{kHz}$ has been chosen. The recorded images (in reflection mode) of fixed HCV29 and T24 cells are shown in Figs. 9 and 10. The scan size was $50 \mu \mathrm{m} \times$ $50 \mu \mathrm{m}$. Topography images clearly show the characteristic shape of each cell type i.e. elongated for HCV29 and oval for T24 cells. In NSOM images, their shape was more pronounced as compared to topography where the cell surface was slightly fuzzy. Moreover, as compared to dried cells, these images showed more features pointing out the regions with different optical properties. 


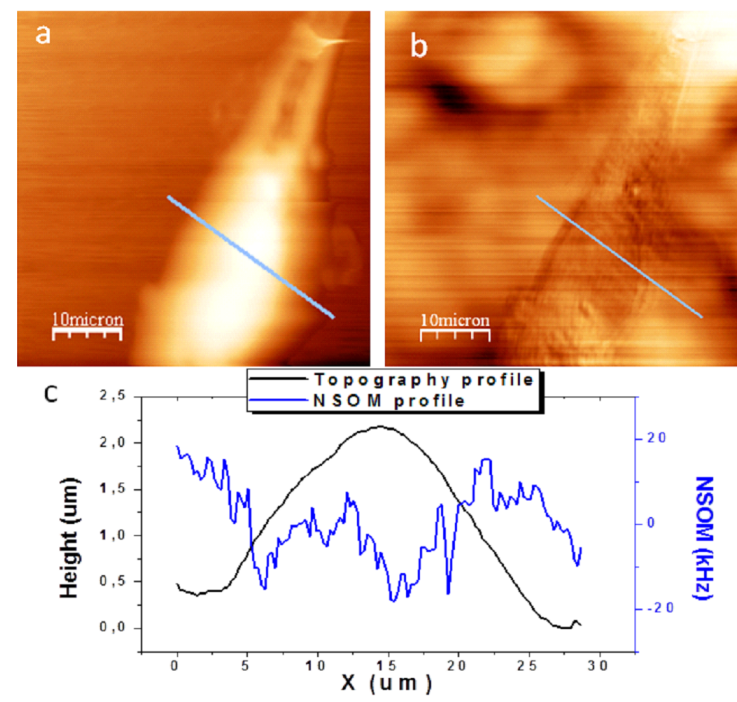

Fig. 9. HCV29 cells in liquid: topography (a), optical (b) images (reflection mode), and profile (c) along the selected line.

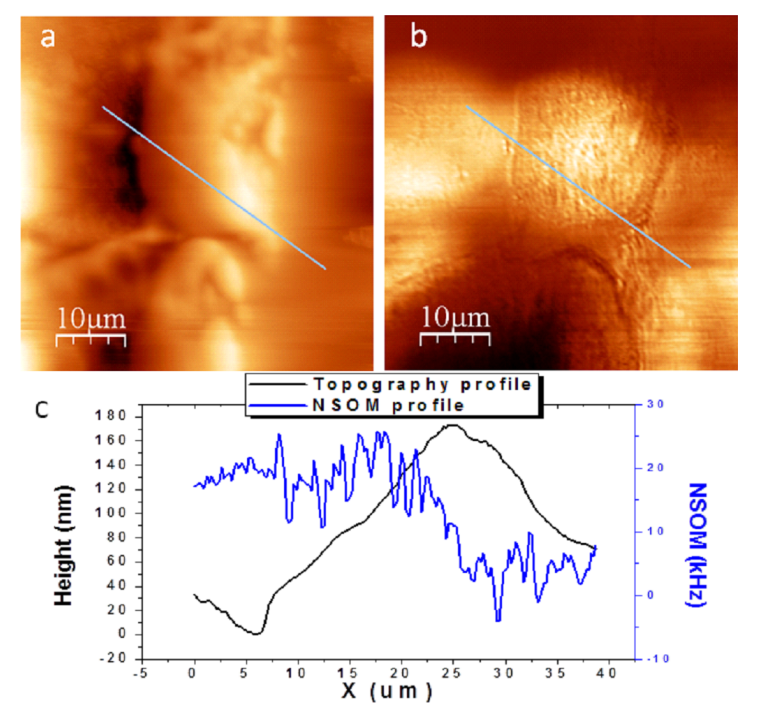

Fig. 10. T24 cells in liquid: topography (a), optical (b) images (reflection mode), and profile (c) along the selected line.

Also, the differences in heights of measured samples indicated changes in the recorded optical signals since they are strongly dependent on the thickness of the cell. Alterations of the NSOM signal can be correlated with different optical properties of the cell membrane and structures inside the cell. The comparison of profiles of the sample height and NSOM signals along selected lines was performed (Figs. 9c and 10c). The height profile, obtained for HCV29 cells, shows only main changes in the cell height while NSOM signal reveals more complex structure within the area of the cell. In the regions, where a small loss of the signal collected by photomultiplier at the cell edges appears, the cell manifests a large change in the NSOM signal as compared to smooth increase or decrease of the cell height. The cross-section profile, obtained for T24 cells, shows the increase of the cell height and simultaneously almost no changes of the optical signal indicating lack of alterations in optical density of T24 cells. On the other hand, the decrease of the NSOM signal is strongly correlated with the decrease of cell height indicating that optically denser materials are detected.

\section{Conclusions}

In the presented studies, successful implementation of transmission and reflection NSOM to biological samples has been demonstrated. Two types of cells (HCV29 and T24) have been studied both in air and liquid conditions, aiming at the characterization of their optical properties. Obtained NSOM optical images are less affected by the tip-surface interactions than topography and phase AFM images. In ambient conditions optical images collected in transmission mode show clearly variations in the optical density of the studied cells, revealing details not visible in AFM images. The NSOM reflection mode is less sensitive since almost no light is reflected from the surface in air. In contrast, it was possible to collect only reflection optical images in liquid environment. Variations of the optical signal can be attributed to the variations of the optical properties of cell membrane and the structures inside the cell. The shape of measured cells recorded by NSOM correlates well with that obtained from AFM imaging (both methods work in non-contact modes), although NSOM tip radius is significantly larger than for typical AFM probe. NSOM imaging can be useful in imaging of biological materials but more reliable methods of tip-sample distance control have to be worked out.

\section{Acknowledgments}

This work was partially supported by the project of the National Science Centre No. 2011/01/N/ST3/02255.

\section{References}

[1] O. Mooren, E. Erickson, N. Dickenson, R. Dunn, J. Lab. Autom., JALA 11, 268 (2006).

[2] R.C. Dunn, Chem. Rev. 99, 2891 (1999).

[3] J. Tersoff, D.R. Hamann, Phys. Rev. B 31, 805 (1985).

[4] G. Binnig, C.F. Quate, Ch. Gerber, Phys. Rev. Lett. 56, 930 (1986).

[5] Y. Martin, H.K. Wickramasinghe, Appl. Phys. Lett. 50, 1455 (1987).

[6] M. Nonnenmacher, M.P. O'Boyle, H.K. Wickramasinghe, Appl. Phys. Lett. 58, 2921 (1991).

[7] E. Betzig, J.K. Trautman, T.D. Harris, J.S. Weiner, R.L. Kostelak, Science 251, 1468 (1991).

[8] T. Berdyyeva, C. Woodwarth, I. Sokolov, Ultramicroscopy 102, 189 (2005). 
[9] D. Allison, N. Mortensen, C. Sullivan, M. Doktycz, WIREs Nanomedicine and Nanobiotechnology 2, 618 (2010).

[10] G. Longo, M. Girasole, G. Pompeo, R. Generosi, M. Luce, A. Cricenti, Phys. Status Solidi B 247, 2051 (2010).

[11] M.F. Garcia-Parajó, B.I. de Bakker, M. Koopman, A. Cambi, F. de Lange, C.G. Figdor, N.F. van Hulst, Nanobiotechnol. 1, 113 (2005).

[12] A.P. Sommer, R.P. Franke, J. Proteome Res. 1, 111 (2002).
[13] T.H. Keller, T. Rayment, D. Klenerman, R.J. Stephenson, Rev. Sci. Instrum. 68, 1448 (1997).

[14] C. Hoppener, J.P. Siebrasse, R. Peters, U. Kubitscheck, A. Naber, Biophys. J. 88, 3681 (2005).

[15] M. De Serio, R. Zenobi, V. Deckert, Trends Anal. Chem. 22, 70 (2003). 